\title{
Intérêts et limites des « indices macrophytes » pour qualifier la mésologie et la physico-chimie des cours d'eau : exemples armoricains, picards et lorrains
}

\author{
J. Hauryl \\ M.-C. Peltre 2 \\ Mots clés : Rivière, indice macrophyte, qualité d'eau, méthodologie, biotypologie.
}

L'estimation de la qualité des cours d'eau, à la fois en terme de milieu physique et de qualité de l'eau, est une nécessité croissante. Les " indices macrophytes " peuvent être utilisés en complémentarité de méthodes plus usuelles, analyses d'eau ou indices biotiques.

Certains indices macrophytes proposés par la littérature ont été appliqués et testés sur 5 rivières du Nord de la France, correspondant à des substrats géologiques et à des situations de pollution et/ou de trophie contrastées. Ce sont : le Diagnostic Phytoécologique à partir des indices d'Ellenberg, le Plant Score de Harding, l'Indice Trophique de Newbold \& Holmes, le Taux de Dommage de Haslam. Une comparaison avec les analyses d'eau a été réalisée.

Les diagnoses sont globalement concordantes, mais leur acuité est variable. Les listes d'espèces fournies par les auteurs sont en général très incomplètes et éludent de nombreux problèmes de taxonomie. Le niveau de bioindication (notion de « trophie », paramètre écologique analytique comme les orthophosphates, ...) en fonction de l'état physique du cours d'eau, est rarement précisé.

Les indices proposés dans les trois premières méthodes citées sont rarement étayés par l'analyse détaillée des distributions espèces/facteurs (profils écologiques). La méthode de Haslam présente un caractère intégrateur marqué fondé sur l'écologie des cours d'eau, même si l'aspect floristique est incomplet. Son application en France est obérée par la méconnaissance des phytocénoses de référence et des séquences de dégradation, et par l'absence d'une grille précise de trophiesubstrat géologique nécessaire pour l'estimation des altérations de la qualité de l'eau. Les méthodes de Harding et de Newbold \& Holmes sont de mise en œuvre plus simple et donnent des indications assez cohérentes.

Malgré ces réserves, la définition d'indices semble intéressante et correspond à une demande des gestionnaires. Des propositions d'amélioration des méthodes existantes, ainsi que des éléments pour l'élaboration d'un indice synthétique sont présentés.

Interest and limits of macrophyte indices to qualify the mesology and the physical chemistry of rivers with examples in Armorica, Picardy and Lorraine

Keywords : River, macrophyte index, water quality, method, biotypology.

There is an ever-increasing need to monitor the quality of watercourses for physical features and water quality. Macrophyte indices may be involved in this, as well as the more usual methods such as water analysis or a benthic invertebrate biotic index.

Some " macrophyte indices » from the literature were applied to 5 rivers in Northern France, differing with respect to geological substratum, trophic level and/or pollution. These methods were : Phytoecological Diagnosis involving Ellenberg's indices, Harding's Plant Score, Newbold \& Holmes's Trophic Index, Haslam's Damage Rating Method. A comparison was made with the results of water analysis.

Diagnoses were convergent, but with differing precision. The floristic lists used by the authors are incomplete and raise many taxonomic queries. The level of bioindication (« trophy ", chemical parameter of pollution,...) with respect to physical features of the watercourse is not often given precisely by the authors. In the case of the methods of Ellenberg, Harding and Newbold \& Holmes, the indices are not based on a detailed study of relationships between ecological factors and plant distribution (" ecological profiles »). Haslam's method is holistic, but the floristic precision is very poor. Its application in France will be difficult because there are many unknown data : reference phytocenosis, degradation successions, precise relationships between geology and water chemistry in each ecoregion. The methods of Harding and Newbold \& Holmes are simpler and lead to quite a good diagnosis.

Nevertheless, macrophyte indices seem interesting and are expected by field engineers. Some improvements for existing methods and general comments for an integrating index are developed.

1. ENSA Botanique et INRA Ecologie hydrobiologique, 65, rue de Saint Brieuc, 35042 Rennes Cedex.

2. Centre de Recherches Ecologiques de l'Université de Metz, B.P. 4116, 57040 Metz Cedex 01.

Texte d'une communication effectuée dans le cadre du Colloque - Limnologie appliquée et application de la Limnologie - Besançon, Franche-Comté, 16-19 novembre 1992. 


\section{Introduction}

La surveillance de l'état des cours d'eau est une nécessité croissante. A côté des analyses physicochimiques habituelles dont le caractère ponctuel est bien connu, des méthodes plus " intégratrices " se sont développées en utilisant les compartiments biologiques, dont les plus connues et les plus utilisées mettent en jeu des invertébrés benthiques.

Les macrophytes (végétaux visibles à l'œil nu regroupant des plantes vasculaires hydrophytes et hélophytes, des bryophytes aquatiques ou supraaquatiques et, par extension, des algues filamenteuses), donnent une image de la qualité de l'eau (Kohler 1975, Whitton 1979, Empain et al. 1980, Mériaux 1982, Haury \& Dutartre 1990). Or ces macrophytes présentent plusieurs intérêts : cartographiables, plus ou moins aisément identifiables, ils permettent d'avoir une vision synthétique et sur le terrain de l'état d'un cours d'eau, orientant les prélèvements pour l'élaboration d'autres diagnoses plus « classiques $\gg$ et confortant leurs résultats.

Pour rendre compte à partir d'un examen in situ des relations existant entre la qualité de l'eau et les macrophytes, trois optiques ont été développées en France :

- la recherche de taxons indicateurs (Mériaux 1982, Haury 1985, 1990a) passe par l'examen de la distribution des espèces en fonction des paramètres analytiques de la qualité de l'eau, la précision du caractère bioindicateur se situant au niveau du jugement d'expert ou du tracé des profils écologiques,

- la mise en œuvre d' " indices macrophytes" qui jusqu'à présent a uniquement fait l'objet de rapports (Léglize \& Peltre 1988, Haury 1989, 1990b, 1991, 1992, Anonyme 1990, 1991, Codhant et al. $1991, \ldots)$,

- le niveau des groupements végétaux a été analysé soit en terme de modifications des associations (Mériaux \& Wattez 1980) soit par l'élaboration d'une " typologie trophique " (Haury 1985) et de séquences d'eutrophisation en milieu calcaire (Carbiener et al. 1990) ou acide (Muller 1990).

Il s'agit présentement d'utiliser certains indices macrophytes élaborés Outre-Manche et d'envisager des modifications à leur apporter. On les appliquera à divers cours d'eau, en fonction des listes floristiques obtenues ou vérifiées par des observateurs confirmés.

\section{Milieux et méthodes d'étude}

\subsection{Cours d'eau, stations étudiées et échantillonnage}

Les cours d'eau considérés sont au nombre de 5 (Fig. 1). Ils ont été retenus, hormis le Tilleul, pour leur homogénéité de substrat géologique. Les abréviations renvoient au Tableau 1 de présentation des stations d'étude. La liste des cours d'eau est donnée d'Est en Ouest, les qualifications trophiques n'étant qu'indicatives :

- la Moselotte est oligotrophe légèrement acide à neutre ; elle coule sur granite, et présente un léger gradient d'eutrophisation essentiellement dû à des rejets urbains à partir de la station M3 ;

- la Petite Seille est eutrophe et se trouve sur des marnes ; elle est barrée par un étang sur la partie amont (entre PS1 et PS2) et reçoit des effluents de petites agglomérations et de petites industries ;

- l'Avre et son affluent, la Noye sont eutrophes, coulent sur calcaires, présentent des pollutions ponctuelles importantes, papeterie pour l'Avre (A2), station d'épuration pour la Noye (N2) dont l'amont a été recalibré (N1M) ;

- le Tilleul, mésotrophe acide, coule sur des granites puis des schistes ; l'amont (T1) subit une pollution ponctuelle importante par une agglomération; des étangs se trouvent entre $\mathrm{T} 1$ et $\mathrm{T} 2$.

Les caractéristiques générales des stations d'étude sont résumées dans le Tableau 1. Pour les cours d'eau lorrains, les stations correspondent au découpage abiotique préconisé par Leglize et al. (1990) ; pour les autres, le choix des stations a été l'étude amont-aval de divers types de pollution.

Les analyses d'eau permettant de juger de la bonne pertinence des indices macrophytes malgré leur caractère ponctuel sont également regroupées dans le Tableau 1.

\subsection{Relevés de végétation}

Les relevés ont été réalisés sur des longueurs suffisantes pour arriver à un " épuisement floristique " de la végétation aquatique, mais aussi des espèces supra-aquatiques en pied de berge ; les longueurs minimales préconisées par Wiegleb (1983) et Haury (1985) sont de $50 \mathrm{~m}$, mais elles ont été plus importantes lorsque les peuplements macrophytiques étaient épars. Les unités transversales étudiées ont été le lit apparent pour les deux cours d'eau lorrains 

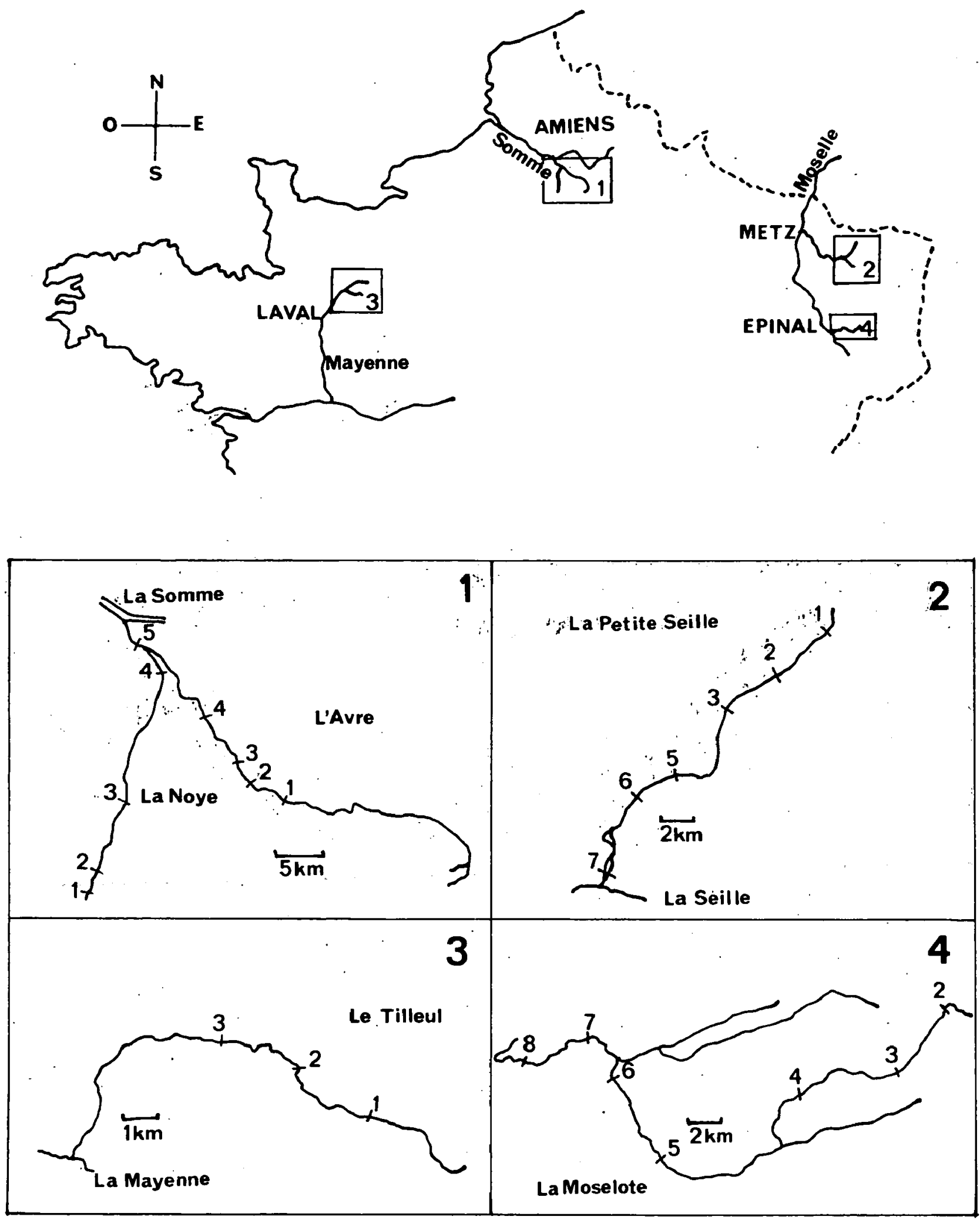

Fig. 1. Localisation des cours d'eau choisis et des stations d'étude.

Fig. 1. Localization of rivers and study sites.

(Léglize \& Grasmuck 1992), le lit et la zone supraaquatique (zone submergée pendant au moins $40 \%$ de l'année selon Holmes \& Whitton 1977) pour les cours d'eau armoricains et picards (Haury 1989, $1990 \mathrm{~b}, 1992)$. Toutes les espèces peu inféodées à l'eau ou qui ne sont pas considérées par l'un des indices sont éliminées des listes. Ces méthodes étant présentées pour la première fois en France, les indices utilisés pour les taxons retenus sont présentés dans le Tableau 2. 
Tableau 1. Caractéristiques des stations d'étude et de la qualité de l'eau.

Table 1. Characteristics of study sites and of water quality.

\begin{tabular}{|c|c|c|c|c|c|c|c|c|c|c|c|c|c|c|}
\hline \multirow{3}{*}{$\begin{array}{l}\text { RIVIERE } \\
\text { GEOLOGIE } \\
\text { STATION }\end{array}$} & \multicolumn{6}{|c|}{ MOSELOTTE } & & \multicolumn{3}{|c|}{ TILLEUL } & \multirow{2}{*}{\multicolumn{3}{|c|}{$\begin{array}{c}\text { NOYE } \\
\text { CALCAIRES }\end{array}$}} & \multirow[b]{3}{*}{ N4 } \\
\hline & \multicolumn{2}{|c|}{ GRANITE } & & & & & \multicolumn{4}{|c|}{ GRANITES/SHISTES } & & & & \\
\hline & M2 & M3 & M4 & M5 & M6 & M7 & M8 & T1 & $\mathrm{T} 2$ & T3 & N1 & N2 & N3 & \\
\hline TEMPERATURE ${ }^{\circ} \mathrm{C}$ & 18 & 17,3 & & 16,7 & 19,2 & 18 & 17,8 & 11,2 & 12,2 & 12,4 & 11,6 & 12,7 & 12,6 & 15 \\
\hline O2 DIssous & 8 & 8 & & 8,4 & 8,9 & 9,3 & 8,4 & 10,1 & 8,2 & 9,8 & & & & \\
\hline$\%$ SAT O2 & 87 & 86 & & 89 & 99 & 101 & 91 & 90 & 76 & 91 & 80 & 69 & 75 & 84 \\
\hline CONDUCTIVITE & 41 & 31 & 54 & 50 & 52 & 50 & 63 & 180 & 190 & 180 & 540 & 560 & 540 & 515 \\
\hline $\mathrm{pH}$ & 6,4 & $6,4^{\circ}$ & 6,7 & 6,5 & 6,5 & 6,3 & 6,3 & 6,8 & 7,1 & 6,9 & 7,3 & 7,3 & 7,6 & 8,2 \\
\hline $\mathrm{NO} 2 \mathrm{mg} \mathrm{N} / \mathrm{I}$ & 0,008 & 0,007 & 0,007 & & 0,01 & 0,01 & 0,007 & 0,02 & 0,02 & 0,016 & $<0,1$ & 0,07 & 0,08 & 0,05 \\
\hline $\mathrm{NO} 3 \mathrm{mg} \mathrm{N} /$ & 0,1 & 0,2 & 0,4 & & 0,1 & 0,5 & 0,5 & 4,1 & 4,5 & 5,7 & 5,9 & 6,6 & 6,4 & 5,45 \\
\hline $\mathrm{NH} 4 \mathrm{mg} \mathrm{N} /$ & 0,04 & $, 0,3$ & 0,17 & & 0,07 & 0,07 & 0,05 & 0,12 & 0,16 & 0,16 & $<0,1$ & 0,18 & $<0,1$ & $<0,1$ \\
\hline PO4 mg P/ & 0,02 & 0,01 & 0,07 & & 0,05 & 0,06 & 0,23 & 0,08 & 0,12 & 0,1 & 0,02 & 0,75 & 0,12 & 0,15 \\
\hline$P$ total $\mathrm{mg} P /$ & 0,1 & 0,2 & 0,2 & & 0,1 & 0,1 & 0,3 & & & & & & & \\
\hline SO4 mg/ & 3 & 3 & 4 & & 21 & 38 & 33,5 & 8 & 8 & 8 & 10,5 & 12,07 & 10,5 & 12 \\
\hline CHLORURES mg/ & 11 & 8,5 & 9,5 & & 9,5 & 10 & 12 & 18 & 17 & 16 & & & & \\
\hline RIVIERE & & & AVRE & & & & PEר & ITE SE & ILLE & & & & & \\
\hline GEOLOGIE & CALC & AIRES & & & & & MARNE & & & & & & & \\
\hline STATION & A1 & $\mathrm{A} 2$ & A3 & A4 & A5 & PS1 & PS2 & PS2b & PS3 & PS4 & PS5 & PS7 & & \\
\hline TEMPERATURE ${ }^{\circ} \mathrm{C}$ & 15,2 & 16,2 & 16,4 & 16,8 & 16,9 & 13,1 & 13,2 & 15,3 & 15,7 & 15,8 & 15,3 & 17,6 & & \\
\hline O2 DIssous & & & & & & 6,3 & 6,6 & 7 & 8,6 & 11,2 & 10,3 & 10,3 & & \\
\hline$\%$ SAT O 2 & 68 & 69 & 12 & 43 & 70 & 62 & 65 & 72 & 89 & 117 & 1.06 & 111 & & \\
\hline CONDUCTIVITE & 640 & 600 & 580 & 595 & 570 & 1700 & 1700 & 1750 & 1900 & 1900 & 1700 & 2300 & & \\
\hline $\mathrm{pH}$ & 7,6 & 7,4 & 7,1 & 7,5 & 7,8 & 7,9 & 7,5 & 7,9 & 7,8 & 7,8 & 7.7 & 7,7 & & \\
\hline $\mathrm{NO} 2 \mathrm{mg} \mathrm{N} / \mathrm{I}$ & 0,096 & 0,02 & 0,21 & 0,12 & 0,09 & 0,11 & 0,19 & 0,16 & 0,15 & 0,19 & 0,09 & 0,29 & & \\
\hline $\mathrm{NO} 3 \mathrm{mg} \mathrm{N} /$ & 4,1 & 3,18 & 2,5 & 1,36 & 3,18 & 2,6 & 3,3 & 3,4 & 3,3 & 3,6 & 3,8 & 1,9 & & \\
\hline $\mathrm{NH} 4 \mathrm{mg} \mathrm{N} /$ & $<0,1$. & $<0,1$ & 0,15 & 0,07 & $<0,1$ & 1,49 & 2,48 & 1,71 & 1,01 & 0,57 & 0,23 & 0,63 & & \\
\hline PO4 mg P/ & 0,26 & 0,03 & 0,13 & 0,2 & 0,16 & 0,85 & 1,72 & 1,35 & 1 & 0,97 & 0,55 & 0,39 & & \\
\hline$P$ total $\mathrm{mg} P /$ & & & & & & 0,9 & 0,6 & 0,2 & 0,9 & 0,9 & 0,5 & 0,8 & & \\
\hline SO4 mg/l & 21 & 28 & 15,5 & 16,5 & 13,5 & 1030 & 956 & 838 & 884 & 925 & 812 & 858 & & \\
\hline CHLORURES mg/ & & & & & & 34,5 & 74 & 60 & 111 & 111,5 & 67,5 & 267 & & \\
\hline
\end{tabular}

Les algues filamenteuses n'ont été identifiées qu'au genre, les autres macrophytes, autant que possible, ont été déterminés jusqu'à l'espèce. L'estimation visuelle des recouvrements a été effectuée par deux observateurs, N. Grasmuck (Université de Metz) et $\mathbf{J}$. Haury qui s'étaient intercalibrés. Les listes floristiques détaillées figurent dans leurs travaux précités.

\subsection{Indices utilisés}

Parmi les indices utilisables, trois prennent en compte la qualité bioindicatrice des espèces : le Diagnostic phytoécologique avec les indices d'Ellenberg (1979), le Plant Score de Harding (1978 in Standing Committee of Analysts (H.M.S.O.) 1987) et l'indice Trophique de Newbold \& Holmes (1987). En revanche, le Taux de Dommage de Haslam (Haslam 1978, Haslam \& Wolseley 1981, Haslam 1987), tout en considérant l'écologie de certains taxons, analyse certains descripteurs de la communauté et préfigure les " indices de communautés ».

\subsubsection{Méthodes basées sur des indices spécifiques}

L'idée majeure est que l'on attribue une valeur aux espèces, lorsque leur valence écologique est suffisamment étroite pour que le chiffre aît une signification. On somme les valeurs d'indices des espèces présentes. Puis en divisant par le nombre d'espèces, on obtient un chiffre moyen qui permet un diagnostic de la station indépendant de la richesse spécifique. 
Tableau 2. Principaux taxons recensés et indices utilisés. - $\mathrm{pH}:$ indices Ellenberg/pH ; $\mathrm{N}$ : indices Ellenberg/Azote ; $\mathrm{S}:$ indices Ellenberg/Sel ; HS : taxons pris en compte par Haslam ( + : taxons confondus ; = : espèces indifférentes à la pollution ; PT : polluotolérantes selon Haslam (échelle croissante) ; HG : indices spécifiques Harding (+ : taxons confondus) ; N\&H : indice Newbold \& Holmes ; $\mathrm{E}$ : taxons euryèces (selon Newbold \& Holmes).

Table 2. Main taxa encountered and indices. - $\mathrm{pH}:$ Ellenberg's indices/pH ; $\mathrm{N}:$ Ellenberg's indices/Nitrogen ; $\mathrm{S}:$ Ellenberg's indices/Salt ; HS : taxa from Haslam's floristic list ( $+:$ Joined taxa ; = : pollution indifferent species) ; PT : pollutiontolerant species following Haslam (increasing scale) ; HG : species index following Harding (+ : Joined taxa) ; N\&H : Newbold \& Holmes' indices ; E : generalist species (following Newbold \& Holmes).

\begin{tabular}{|lllllllll}
\hline TAXONS / INDICES & $\ldots$ & $\ldots$ & pH & N & S & HS & PT & HG \\
\hline
\end{tabular}

Algues
Batrachospermum sp.
Chaetophora sp.
Chara sp
Cladophora sp.
Closterium sp.
Diatomées filamenteuses
Enteromorpha sp.
Lemanea sp.
Microspora sp.
Nitella sp.
Oedogonium sp.

Oscillatoria sp.

Placage de cyanophycées

Rhizoclonium sp.

Spirogyra sp.

Stigeoclonium sp.

Ulothrix sp.

Vaucheria sp

Lichen

Dermatocarpon fuviatile

Bryophytes

Brachythecium rivulare B.e.

Brachythecium sp.

Chiloscyphus pallescens (Ehrh.) Dum.

Chiloscyphus polyanthus (L.) Corda

Conocephalum conicum (L.) Dum

Fissidens crassipes Wils.

Fissidens pusillus Wils.

Fontinalis antipyretica Hedw.

Fontinalis squamosa Hedw.

Hygroamblystegium fluviatile (Sm.) Loeske

Hyocomium armoricum (Brid.) Wijk \& Marg.

Leptodictyum riparium (Hedw.) Warnst.

Lunularia cruciata (L.).Dum.

Marchantia polymorpha L.

Pellia fabbroniana Raddi.

Pellia epiphylla (L.) Corda

Platyphypnidium riparioüdes (Hedw.) Dix.

Porella pinnata L.

Rhacomitrium aciculare (Hedw) B. e.

Riccia fluitans L.

Scapania undulata (L.) Dum.

Schistidium rivulare (Brid.) Deg.

Ptéridophytes

Azolla filiculoïdes Lam.

Phanérogames hydrophytes

Callitriche hamulata Kütz

Callitriche obtusangula Le Gall

Callitriche platycarpa Kütz

Callitriche stagnalis Scop.

Ceratophyllum demersum $\mathrm{L}$.

Elodea canadensis Michx

Lemna gibba L

Lemna minor $\mathrm{L}$.

$\begin{array}{ccccc}1 & 4 & & \\ & & 7 & & \\ 1+ & 4+ & & & \\ 1+ & 4+ & 3 & 112 & \\ & & & & \\ 1 & & 3 & 136 & \mathrm{X} \\ & & 8 & 51 & \\ 1+ & 4+ & & & \\ 1+ & & & & \end{array}$

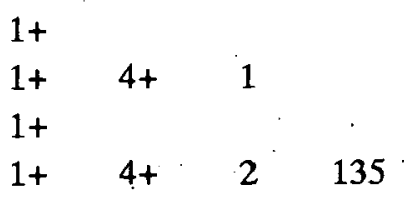

1

$1+$

$1+$

$1+\quad 10$

$1+10$

$1+$

$1+\quad 10$

$1+\quad 10$

$1+$

$1+$

56

40

6

10

2

126

- 25

$50 \mathrm{X}$

10

10

18

25

80

X $\quad 8 \quad \cdots 0$

23

$\begin{array}{llll}X & 6 & 0 & 1\end{array}$

$6+$

$6+83$

$\begin{array}{llll}\mathrm{X} & 6 & 0 & 1+\end{array}$

$\begin{array}{llll}X & 6 & 0 & 1+\end{array}$

$\begin{array}{llll}\mathrm{X} & 6 & 0 & 1+\end{array}$

$\begin{array}{llll}\mathrm{X} & 6 & 0 & 1+ \\ \mathrm{X} & 8 & 0 & 1\end{array}$

$\begin{array}{lll}8 & 0\end{array}$

$\begin{array}{llll}7 & 8 & 0 & 1+\end{array}$

X $\quad$ X $01+$ 
Tableau 2. (suite).

Table 2. (continuation).

\begin{tabular}{|llllllllll}
\hline TAXONS / INDICES & pH & N & S & HS & PT & HG & N\&H & E \\
\hline
\end{tabular}

Lemna trisulca L..

Myriophyllum spicatum L.

Nuphar lutea (L.) Sibth \& Sm

Potamogeton crispus $\mathrm{L}$.

Potamogeton pectinatus L

Potamogeton perfoliatus $\mathrm{L}$.

Ranunculus peltatus Schrank.

Ranunculus penicillatus (Dum.) Bab.

Sagittaria sagittifolia L.

Sparganium emersum Rehmann

Spirodela polyrhiza (L.) Schleiden

Zannichellia palustris (L.)

Herbacées hélophytes

Acorus calamus L.

Alisma plantago $\mathrm{L}$.

Apium nodiflonum Lag.

Berula erecta (Hudson) Coville

Caltha palustris $\mathrm{L}$.

Carex acuta $(=C$. gracilis $) \mathrm{L}$.

Carex paniculata $\mathrm{L}$.

Carex riparia Curtis

Carex vesicaria $\mathrm{L}$

Galium palustre L.

Glyceria fluitans (L.) R. Br

Hydrocotyle vulgaris $\mathrm{L}$.

Iris pseudacorus $\mathrm{L}$.

Juncus bulbosus L.

Mentha aquatica $\mathrm{L}$.

Montia fontana $\mathrm{L}$.

Myosotis gr. scorpioides L.

Myosoton aquaticum (L.) Moench

Nasturtium officinale $\mathrm{R}$. Br.

Oenanthe aquatica (L.) Poiret.

Oenanthe crocata $\mathrm{L}$.

Phalaris arundinacea $\mathrm{L}$.

Phragmites communis

Polygonum amphibium $\mathrm{L}$.

Polygonum hydropiper L.

Ranunculus flammula $\mathrm{L}$.

Rumex hydrolapathum Hudson

Scutellaria galericulata L.

Sparganium erectum $\mathrm{L}$.

Typha latifolia L.

Veronica anagallis-aquatica $\mathrm{L}$.

Veronica beccabunga L.

Phanérophyte subaquatique

Solanum dulcamara $\mathrm{L}$

Herbacées hygro \& mésophiles

Agrostis stolonïfera L

Epilobium hirsutum $\mathrm{L}$.

Juncus acutiflorus Ehrh.

Juncus bufonius L.

Juncus conglomeratus $\mathrm{L}$.

Juncus effusus L.

Scirpus sylvaticus L.

$\begin{array}{cccccccc}7 & 6 & 0 & 1 & & 4+ & 89 \\ 8 & \text { X } & \text { I } & 1 & 2 & 7 & 148 & \\ 6 & \text { X } & 0 & 1 & 4 & 6 & 138 & \\ 7 & 6 & 0 & 1 & 4 & 4 & 137 & \\ 7 & 7 & \text { I } & 1 & 4 & 1 & 149 & \\ 7 & 4 & \text { I } & & & 7 & 135 & \\ 4 & 4 & 0 & 1+ & =+ & 8 & 48 & \text { X } \\ \text { X } & \text { X } & \text { X } & 1+ & =+ & 6 & 69 & \\ 7 & 6 & 0 & 1 & & 4 & 127 & \\ \text { X } & 5 & 0 & 1 & = & 3 & 102 & \\ \text { X } & 7 & 0 & 1 & & 4+ & & \\ 7 & 6 & \text { II } & 1 & & 5 & 150 & \end{array}$

$\begin{array}{lll}7 & 7 & 0\end{array}$

$\begin{array}{lllllll}\mathrm{X} & 8 & 0 & 1 & 3 & 109 & \mathrm{X}\end{array}$

$\begin{array}{llllll}X & 6 & I & 1 & = & 4\end{array}$

$\begin{array}{lllll}\mathrm{X} & 7 & \mathrm{I} & 1 & 81\end{array}$

$\begin{array}{lllllll}X & X & 0 & 1 & 6 & 54 & X\end{array}$

$\begin{array}{lllllll}6 & 4 & 0 & 1+ & \text { * }^{*} & 8+ & 40\end{array}$

$94 \quad 0 \quad 2 \quad=^{*} 8+113$

$7.4 \quad 0 \quad 1+\quad=^{*} \quad 8+114$

$6 \quad 5 \quad 0.1+\quad=^{*} \cdot 8+$

$\mathrm{X} 40$.

$\mathrm{X} \quad 7 \quad 0$

$2 \quad 20$

X 70$$
\mathbf{X}
$$ 
- Diagnostic phytoécologique

Ellenberg (1979) a défini l'écologie d'un grand nombre de plantes vasculaires médioeuropéennes vis-à-vis de toute une série de facteurs, dont nous retiendrons le $\mathrm{pH}$, l'azote et le sel. Les résultats pour chaque paramètre correspondent à la moyenne des indices des espèces ayant une note pour ce facteur. Néanmoins pour le sel, le calcul est réalisé non seulement avec les halophytes, mais aussi avec toutes les autres espèces qui sont supposées non résistantes au sel.

\section{- Plant Score de Harding}

Par rapport à la liste précédente théoriquement exhaustive pour les plantes vasculaires, Harding considère une liste « autorisée » de 74 taxons qui suppose une bonne acuité de détermination. Les indices spécifiques, compris entre 1 et 10 , sont censés traduire la « qualité de l'eau ». L'auteur donne des indications pour quelques taxons qu'il n'a pas considérés car absents du Nord-Est de l'Angleterre où il a travaillé. Dans les calculs, nous appliquerons ses directives.

Le Plant Score correspond à la somme des indices spécifiques ; il peut varier de 0 (absence de végétation macrophytique) à plus de 100 (végétation diversifiée). Pour ne pas survaloriser la richesse spécifique, et donc la zonation amont-aval, la moyenne (Score Index Par Taxon) est calculée.

\section{- Indices Trophiques}

Les Indices Trophiques fonctionnent un peu comme le Score Index par Taxon de Harding, mais les 150 espèces prises en compte sont hiérarchisées selon un gradient de trophie, de 1 à 150. Ce classement correspond à une analyse multidimensionnelle réalisée sur plus de 1000 relevés. L'Indice Trophique total est la somme des indices spécifiques divisée par le nombre d'espèces. Toutefois, dans le calcul, les auteurs préconisent de ne pas prendre en compte les espèces à large amplitude écologique, ce qui donne un Indice Trophique des sténoèces.

\subsubsection{Taux de Dommage}

La méthode des dommages part d'une double constatation :

- toutes les espèces présentes n'ont pas la même signification écologique ;

- les caractères généraux de la végétation peuvent être comparés à une phytocénose « en équilibre » adaptée aux conditions morphométriques et hydrogéologiques des cours d'eau.

Là encore, la méthode prend en considération une liste d'espèces « autorisées 》. Cette liste (70 taxons - Haslam 1987) amalgame ou élimine tous les taxons dont la détermination est difficile : renoncules, callitriches, mousses, algues filamenteuses, ... Haslam (1987) décrit des phytocénoses, notamment pour la France, avec liste et richesse floristiques, recouvrement et catégorie trophique (déterminée par la géologie et l'importance du cours d'eau), qui servent de référence pour le calcul des Taux de Dommage.

Pour chaque station, des indices analytiques « de dommage " sont calculés selon les paramètres suivants : faible richesse spécifique, écart de cette dernière avec la référence, présence de taxons polluotolérants, recouvrements d'《 espèces spéciales ", Potamogeton pectinatus et algues filamenteuses vertes, réduction du recouvrement par rapport à la " normale ", changement de catégorie trophique (estimée par l'appartenance des espèces à une catégorie trophique).

La somme de ces indices partiels définit un « Taux de Dommage ", compris entre 0 et plus de 22, traduisant une altération par rapport à l'optimum, cette altération correspondant à une dégradation de la qualité de l'eau et/ou à des modifications du milieu physique.

\section{Résultats et interprétation}

\subsection{Résultats généraux}

Les résultats des calculs, ainsi que les éléments analytiques intermédiaires sont résumés dans le Tableau 3. Les variations des indices sont illustrées sur la Figure 2. Les corrélations entre méthodes donnent les cœefficients de détermination $\mathrm{r}^{2}$ suivants : 0,36 entre Haslam et Harding, 0,43 entre Haslam et Newbold \& Holmes (Indice Trophique moyen des sténoèces) et 0,49 entre Harding et Newbold \& Holmes (pour les 2 Indices Trophiques moyens).

Pour le Diagnostic Phytoécologique, les nombres d'espèces ayant un ou des indices pour au moins l'un des facteurs considérés sont compris entre 4 et 63 . Ceci se traduit par des absences de renseignements concernant notamment le $\mathrm{pH}$. Les notes moyennes de $\mathrm{pH}$ sont comprises entre 4,5 et 7,5 ; celles de l'azote varient entre 4 et 7,1 ; pour le sel, les notes moyennes sont comprises entre 0 et 0,25 pour l'ensemble des espèces. 


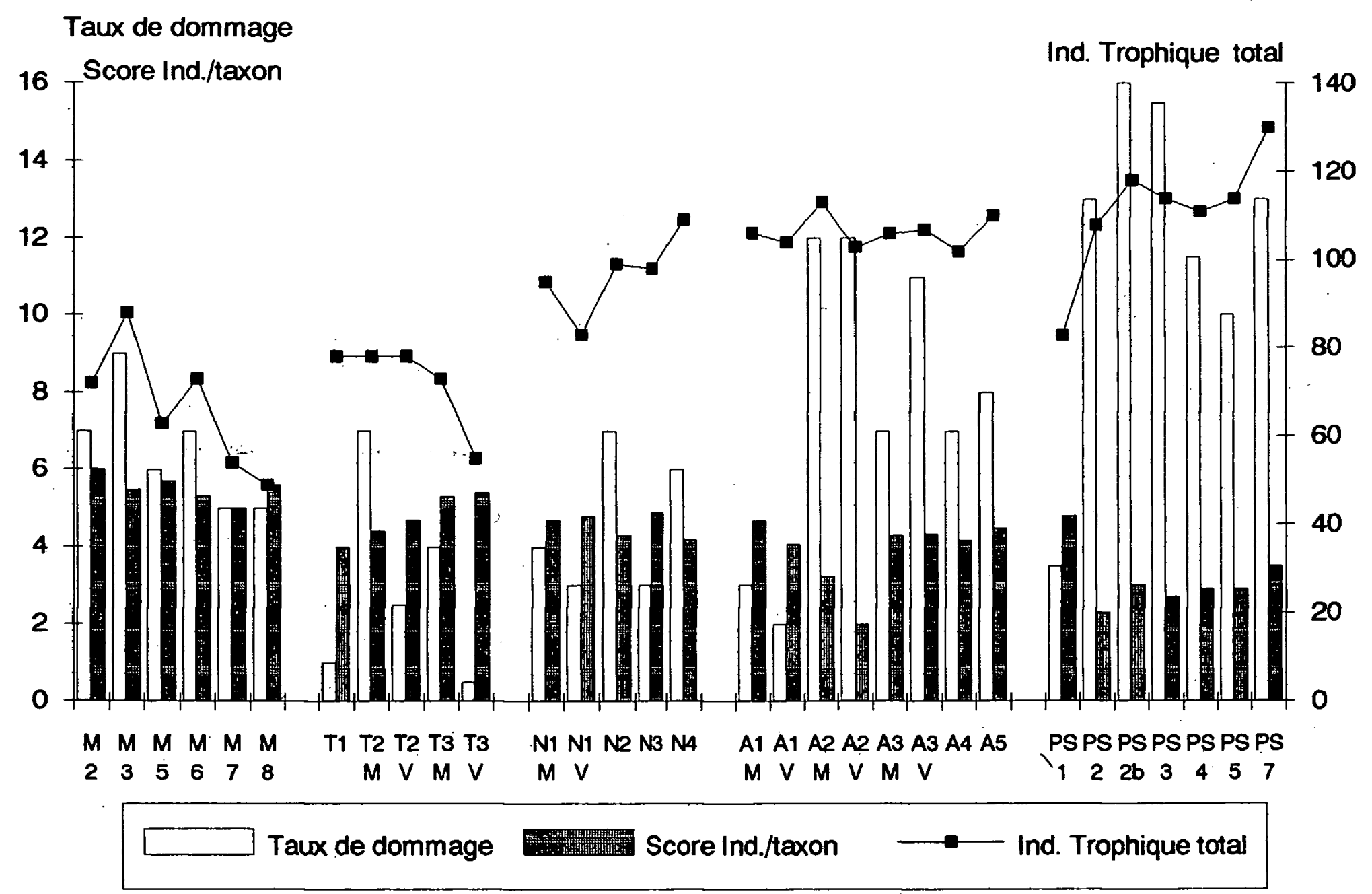

Fig. 2. Evolution des indices sur les cours d'eau retenus. - M : Moselotte ; $T:$ Tilleul ; N : Noye ; A : Avre : PS : Petite Seille. Fig. 2. Indices changes for study watercourses. $-M$ : Moselotte ; $T$ : Tilleul ; $:$ Noye ; A : Avre ; PS : Petite Seille.

Pour le Plant Score, le nombre d'espèces varie entre 3 et 10 pour les cours d'eau lorrains, entre 6 et 34 pour ceux de l'Ouest. Les Plant Score sont compris entre 7 et 179 , ce qui donne des Score Index Par Taxon compris entre 2,00 et 8,00 . L'échantillon ne comprend donc pas de cours d'eau totalement dégradé ni totalement indemne de pollution.

Pour les Indices Trophiques, le nombre total des espèces considérées est compris entre 3 et 25 ( 3 à 11 pour les cours d'eau lorrains, 6 à 25 pour les autres) ; pour les seules espèces sténoèces, les extrêmes sont de 1 à 19. Les Indices Trophiques total et des sténoèces varient respectivement entre 54 et 118 , et entre 46 et 149. Pour cet indice, la dernière gamme de valeurs montre que si les cours d'eau très oligotrophes sont absents de l'échantillon, le maximum de trophie est presque atteint.

Pour le Taux de Dommage, le nombre de taxons pris en considération est compris entre 2 et 21 ( 2 à 7 pour les cours d'eau lorrains, 5 à 21 pour les autres). Les Taux de Dommage varient entre 1 et $16:$ la dégradation maximale n'est donc pas représentée dans l'échantillon.

\subsection{Relations entre indices macrophytes et qualité des cours d'eau}

\subsubsection{Des situations différentes selon les cours d'eau}

Les situations sont nettement contrastées selon les cours d'eau : il semble donc possible de retrouver la typologie établie a priori selon la trophie des eaux et la géologie.

Ainsi, la Petite Seille, l'Avre, la Noye, cours d'eau marneux ou calcaires « eutrophes », présentent des Score Index Par Taxon en général inférieurs à $5(2,00$ à 4,91), contrairement à la Moselotte, plutôt « oligotrophe » $(5,00$ à-8,00); le Tilleul, « mésotrophe " a une position intermédiaire $(4,00$ à 5,44$)$. 
Tableau 3. Résultats des indices macrophytes. - $\mathrm{M}$ : Moselotte ; $\mathrm{T}:$ Tilleul ; N : Noye ; A : Avre ; PS : Petite Seille. Ligne « Cat. trophique attendue $"$ : Code des couleurs de Haslam selon un gradient croissant de trophie : $\mathrm{J}:$ jaune ; V : vert ; T : turquoise ; $P$ : pourpre.

Table 3. Macrophyte index results. - $\mathrm{M}$ : Moselotte ; $\mathrm{T}$ : Tilleul ; N : Noye ; A : Avte ; PS : Petite Seille. Lign « Cat. trophique attendue » : Colour banding from Haslam (increasing trophy scale) : $\mathrm{J}:$ yellow ; $\mathrm{V}:$ green $; \mathrm{T}:$ turquoise ; $\mathrm{P}:$ purple.

\begin{tabular}{lllllllllllllll}
\hline STATIONS & M2 & M3 & M5 & M6 & M7 & M8 & T1 & T2M & T2V & T3M T3V N1M N1V & N2 & N3 & N4 \\
METHODES ET RESULTATS & & & & & & & & & & & & & &
\end{tabular}

$\begin{array}{lllllllllllllllll}\text { NOMBRE TOTAL DE TAXONS } & 10 & 7 & 15 & 8 & 15 & 7 & 44 & 64 & 53 & 62 & 40 & 11 & 16 & 16 & 17 & 22\end{array}$

METHODE HASLAM

INDICES ANALYTIQUES

Richesse spécifique

Chute de diversité

Espèces polluotolérantes

Chute de recouvrement

Catégorie trophique

Espèces spéciales

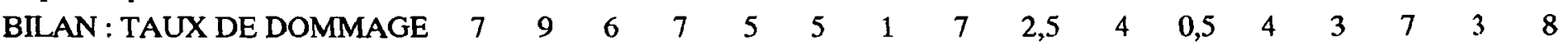

ELEMENTS/METHODE HASLAM

Phytocénoses de référence

$\begin{array}{lcccccccccccccccc}\text { Nombre d'espèces attendu } & 2 & 3 & 3 & 3 & 3 & 3 & 5 & 5 & 5 & 5 & 5 & 7 & 7 & 7 & 7 & 8 \\ \text { Recouvrement attendu } & 10 & 10 & 10 & 10 & 10 & 10 & 40 & 40 & 40 & 40 & 40 & 80 & 80 & 80 & 80 & 60 \\ \begin{array}{l}\text { Catégorie trophique attendue } \\ \text { Phytocénoses observées }\end{array} & \mathrm{J}-\mathrm{V} & \mathrm{V} & \mathrm{V} & \mathrm{V} & \mathrm{V} & \mathrm{V} & \mathrm{T} & \mathrm{T} & \mathrm{T} & \mathrm{T} & \mathrm{T} & \mathrm{T} & \mathrm{T} & \mathrm{T} & \mathrm{T} & \mathrm{T} \\ \text { Nombre d'espèces } & & & & & & & & & & & & & & & \\ \text { Nb de polluotolérantes } & 2 & 2 & 4 & 2 & 4 & 3 & 13 & 18 & 13 & 15 & 9 & 5 & 9 & 5 & 7 & 11 \\ \text { \% de polluotolérantes } & 0 & 0,5 & 1,5 & 0 & 1,5 & \mathbf{0 , 5} & 4,5 & 4 & \mathbf{3} & \mathbf{3 , 5} & 2,5 & \mathbf{0} & 1,5 & 1 & 1 & 4,25 \\ \text { Recouvrement observé } & 0,00 & 0,25 & 0,38 & \mathbf{0 , 0 0} & 0,38 & 0,17 & \mathbf{0 , 3 5} & 0,22 & 0,23 & \mathbf{0 , 2 3} & 0,28 & 0,00 & 0,17 & 0,20 & 0,14 & 0,39 \\ & 5 & 1 & 15 & 5 & 40 & 5 & 80 & 15 & 25 & 10 & 40 & 95 & 20 & 30 & 95 & 70\end{array}$

\section{DLAGNOSTIC /ELLENBERG}

Nombre total d'espèces

Nombre d'espèces /pH

Total pH

Note moyenne $/ \mathrm{pH}$

Nombre d'espèces $/ \mathbf{N}$

Total N

Note moyenne $\mathbf{N}$

Nombre d'espèces /sel

Total /sel

Note moyenne halophytes

Note moyenne/sel

\section{METHODE HARDING}

Nombre d'espèces

PLANT SCORE

SCORE INDEX PAR TAXON

$\begin{array}{cccccccccccccccc}4 & 4 & 3 & 3 & 3 & 3 & 0 & 0 & \because 0 & 0 & 0 & 2 & 0 & 2 & 1 & 0 \\ 0 & 1 & 0 & 1 & 0 & 0 & 0 & 0 & 0 & 0 & 0 & 2 & 0 & 2 & 0 & 0 \\ 0 & 0 & 1 & 0 & 1 & 0 & 1 & 0 & 0 & 0 & 0,5 & 0 & 0 & 0 & 0 & 1 \\ 2 & 4 & 0 & 2 & 0 & 2 & 0 & 3 & 1,5 & 3 & 0 & 0 & 3 & 3 & 0 & 0 \\ 1 & 0 & 0 & 0 & 1 & 0 & 0 & 2 & 1 & 1 & 0 & 0 & 0 & 0 & 0 & 3 \\ 0 & 0 & 2 & 0 & 0 & 0 & 0 & 2 & 0 & 0 & 0 & 0 & 0 & 0 & 2 & 4 \\ 7 & 9 & 6 & 7 & 5 & 5 & 1 & 7 & 2,5 & 4 & 0,5 & 4 & 3 & 7 & 3 & 8\end{array}$

\section{METHODE HOLMES \& NEWBOLD}

Nombre total d'espèces

TOTAL

INDICE TROPHIQUE TOTAL

$\mathrm{Nb}$ d'espèces sténoèces

TOTAL STENOECES

IND. TROPHIQUE STENOECES

$\begin{array}{cccccccccccccccc}7 & 5 & 14 & 8 & 12 & 7 & 42 & 63 & 52 & 59 & 38 & 11 & 16 & 16 & 16 & 22 \\ 2 & 0 & 2 & 1 & 2 & 0 & 16 & 23 & 16 & 20 & 12 & 4 & 5 & 4 & 3 & 13 \\ 10 & 0 & 14 & 5 & 12 & 0 & 72 & 110 & 76 & 98 & 64 & 30 & 37 & 29 & 21 & 94 \\ 5,00 & ? & 7,00 & 5,00 & 6,00 & ? & 4,50 & 4,78 & 4,75 & 4,90 & 5,33 & 7,50 & 7,40 & 7,25 & 7,00 & 7,23 \\ 3 & 2 & 6 & 3 & 5 & 3 & 29 & 39 & 33 & 36 & 21 & 9 & 12 & 9 & 8 & 16 \\ 15 & 11 & 39 & 17 & 31 & 19 & 162 & 211 & 179 & 195 & 113 & 60 & 81 & 61 & 52 & 102 \\ 5,00 & 5,50 & 6,50 & 5,67 & 6,20 & 6,33 & 5,59 & 5,41 & 5,42 & 5,42 & 5,38 & 6,67 & 6,75 & 6,78 & 6,50 & 6,38 \\ 0 & 0 & 0 & 0 & 0 & 0 & 3 & 3 & 2 & 2 & 1 & 1 & 2 & 2 & 2 & 4 \\ 0 & 0 & 0 & 0 & 0 & 0 & 3 & 3 & 2 & 2 & 1 & 1 & 2 & 2 & 2 & 4 \\ ? & ? & ? & ? & ? & ? & 1 & 1 & 1 & 1 & 1 & 1 & 1 & 1 & 1 & 1\end{array}$

$\begin{array}{lllllllllllllllll}0,00 & 0,00 & 0,00 & 0,00 & 0,00 & 0,00 & 0,07 & 0,05 & 0,04 & 0,03 & 0,03 & 0,09 & 0,13 & 0,13 & 0,13 & 0,18\end{array}$ $\begin{array}{llllllllllllllll}4 & 4 & 7 & 3 & 6 & 5 & 24 & 32 & 27 & 34 & 18 & 6 & 9 & 11 & 11 & 14\end{array}$ $\begin{array}{llllllllllllllll}32 & 22 & 40 & 16 & 30 & 28 & 96 & 141 & 128 & 179 & 98 & 28 & 43 & 47 & 54 & 59\end{array}$

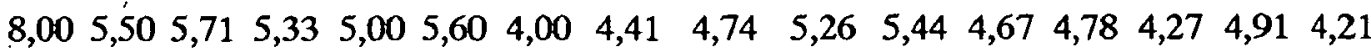

\begin{tabular}{cccccccccccccccc}
3 & 4 & 8 & 4 & 6 & 5 & 19 & 27 & 22 & 25 & 11 & 6 & 8 & 8 & 10 & 16 \\
215 & 350 & 507 & 291 & 324 & 246 & 1481 & 2115 & 1722 & 1826 & 606 & 567 & 661 & 789 & 978 & 1748 \\
72 & 88 & 63 & 73 & 54 & 49 & 78 & 78 & 78 & 73 & 55 & 95 & 83 & 99 & 98 & 109 \\
2 & 1 & 4 & 2 & 3 & 3 & 7 & 12 & 8 & 9 & 3 & 3 & 3 & 4 & 7 & 11 \\
161 & 110 & 183 & 92 & 143 & 143 & 624 & 929 & 624 & 590 & 116 & 256 & 326 & 441 & 655 & 1235 \\
81 & 110 & 46 & 46 & 48 & 48 & 89 & 77 & 78 & 66 & 39 & 85 & 109 & 110 & 94 & 112 \\
\hline
\end{tabular}


Tableau 3. (suite).

Table 3. (continuation).

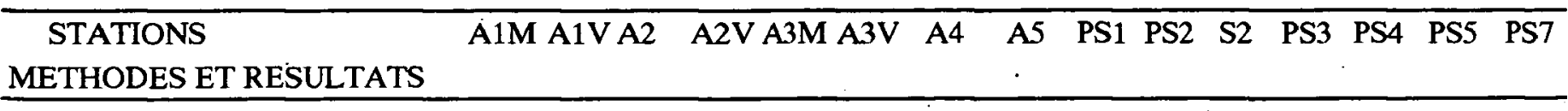

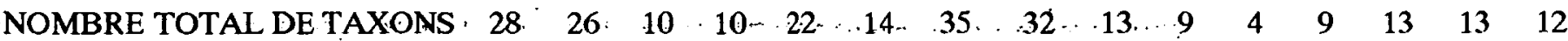

METHODE HASLAM

INDICES ANALYTIQUES

$\begin{array}{lcccccccccccccccc}\text { Richesse spécifique } & 0 & 0 & 1 & 2 & 0 & 1 & 0 & 0 & 1 & 3 & 4 & 3 & 2 & 2 & 2 \\ \text { Chute de diversité } & 0 & 0 & 2 & 4 & 0 & 2 & 0 & 0 & 0 & 4 & 5 & 4 & 2 & 2 & 2 \\ \text { Espèces polluotolérantes } & 0 & 0 & 0 & 1 & 0 & 1 & 1 & 1 & 0,5 & 2 & 3 & 3 & 2 & 2 & 2 \\ \text { Chute de recouvrement } & 0 & 1 & 3 & 0 & 0 & 3 & 0 & 1 & 0 & 0 & 2 & 1,5 & 1,5 & 0 & 1 \\ \text { Catégorie trophique } & 1 & 1 & 4 & 4 & 3 & 3 & 4 & 4 & 0 & 0 & 0 & 0 & 0 & 1 & 2 \\ \text { Espèces spéciales } & 2 & 0 & 2 & 1 & 4 & 1 & 2 & 2 & 2 & 4 & 2 & 4 & 4 & 3 & 4 \\ \text { BILAN : TAUX DE DOMMAGE } & 3 & 2 & 12 & 12 & 7 & 11 & 7 & 8 & 3,5 & 13 & 16 & 16 & 11,5 & 10 & 13 \\ \text { ELEMENTS/METHODE HASLAM } & & & & & & & & & & & & & \end{array}$

Phytocénoses de référence

Nombre d'espèces attendu

Recouvrement attendu

$\begin{array}{llllllllllllllll}9 & 9 & 9 & 9 & 9 & 9 & 9 & 9 & 7 & 7 & 7 & 7 & 7 & 8 & 8\end{array}$

Catégorie trophique attendue

$\begin{array}{lllllllllllllll}80 & 80 & 80 & 80 & 80 & 80 & 80 & 80 & 80 & 80 & 80 & 80 & \cdot 80 & 80 & 80\end{array}$

Phytocénoses observées

Nombre d'espèces

$\begin{array}{lllllllllllll}\mathrm{T} & \mathrm{T} & \mathrm{T} & \mathrm{T} & \mathrm{T} & \mathrm{T} & \mathrm{T} & \mathrm{T} & \mathrm{T}(\mathrm{P}) \mathrm{T}(\mathrm{P}) \mathrm{T}(\mathrm{P}) & \mathrm{T}(\mathrm{P}) \mathrm{T}(\mathrm{P}) & \mathrm{T}-\mathrm{P} & \mathrm{T}-\mathrm{P}\end{array}$

$\mathrm{Nb}$ de polluotolérantes

$\begin{array}{llllllllllllllll}12 & 11 & 7 & 5 & 10 & 7 & 21 & 19 & 7 & 3 & 2 & 3 & 5 & 6 & 6\end{array}$

$\%$ de polluotolérantes

$\begin{array}{lllllllllllllll}3 & 2,5 & 1 & 2 & 1,5 & 2,5 & 7 & 5,75 & 2 & 2 & 1,5 & 2,5 & 3 & 3,5 & 4\end{array}$

Recouvrement observé

$\begin{array}{lllllllllllllll}0,25 & 0,23 & 0,14 & 0,40 & 0,15 & 0,36 & 0,33 & 0,30 & 0,29 & 0,67 & 0,75 & 0,83 & 0,60 & 0,58 & 0,67\end{array}$

$\begin{array}{lllllllllllllll}70 & 60 & 20 & 90 & 70 & 30 & 80 & 60 & 90 & 80 & 40 & 50 & 50 & 90 & 60\end{array}$

DIAGNOSTIC /ELLENBERG

Nombre total d'espèces

Nombre d'espèces /pH

Total pH

Note moyenne $/ \mathrm{pH}$

Nombre d'espèces $/ \mathrm{N}$

Total $\mathbf{N}$

Note moyenne $/ \mathbf{N}$

Nombre d'espèces /sel

Total /sel

Note moyenne halophytes

Note moyenne /sel

$\begin{array}{ccccccccccccccc}27 & 24 & 9 & 9 & 20 & 14 & 33 & 30 & 10 & 7 & 4 & 8 & 11 & 12 & 11 \\ 11 & 11 & 3 & 4 & 9 & 7 & 16 & 15 & 4 & 2 & 1 & 2 & 5 & 5 & 5 \\ 78 & 75 & 22 & 29 & 65 & 48 & 111 & 103 & 28 & 14 & 7 & 14 & 32 & 35 & 35 \\ 7,09 & 6,82 & 7,33 & 7,25 & 7,22 & 6,86 & 6,94 & 6,87 & 7,00 & 7,00 & 7,00 & 7,00 & 6,40 & 7,00 & 7,00 \\ 20 & 19 & 7 & 7 & 17 & 11 & 27 & 25 & 7 & 3 & 2 & 3 & 6 & 8 & 4 \\ 137 & 122 & 45 & 50 & 112 & 73 & 181 & 165 & 47 & 20 & 14 & 21 & 37 & 52 & 25 \\ 6,85 & 6,42 & 6,43 & 7,14 & 6,59 & 6,64 & 6,70 & 6,60 & 6,71 & 6,67 & 7,00 & 7,00 & 6,17 & 6,50 & 6,25 \\ 5 & 4 & 2 & 1 & 3 & 1 & 3 & 5 & 1 & 1 & 1 & 1 & 1 & 2 & 2 \\ 6 & 5 & 2 & 1 & 3 & 1 & 3 & 5 & 1 & 1 & 1 & 1 & 1 & 3 & 2 \\ 1,2 & 1,25 & 1 & 1 & 1 & 1 & 1 & 1 & 1 & 1 & 1 & 1 & 1 & 1,5 & 1 \\ 0,19 & 0,17 & 0,22 & 0,11 & 0,15 & 0,07 & 0,09 & 0,17 & 0,10 & 0,14 & 0,25 & 0,13 & 0,09 & 0,17 & 0,18\end{array}$

METHODE HARDING

$\begin{array}{lllllllllllllllll}\text { Nombre d'espèces } & 12 & 14 & 8 & 6 & 10 & 9 & 23 & 21 & 6 & 3 & 4 & 6 & 8 & 9 & 10\end{array}$

PLANT SCORE

$\begin{array}{lllllllllllllll}56 & 57 & 26 & 12 & 43 & 39 & 96 & 94 & 29 & 7 & 12 & 16 & 23 & 26 & 35 .\end{array}$

$\begin{array}{llllllllllllllllllll}\text { SCORE INDEX PAR TAXON } \quad 4,67 & 4,07 & 3,25 & 2,00 & 4,30 & 4,33 & 4,17 & 4,48 & 4,83 & 2,33 & 3,00 & 2,67 & 2,88 & 2,89 & 3,50\end{array}$

\section{METHODE HOLMES \& NEWBOLD}

Nombre total d'espèces

TOTAL

INDICE TROPHIQUE TOTAL

$\mathrm{Nb}$ d'espèces sténoèces

TOTAL STENOECES

IND. TROPHIQUE STENOECES $\begin{array}{lllllllllllllll}16 & 15 & 7 & 7 & 14 & 11 & 25 & 24 & 8 & 4 & 4 & 6 & 9 & 11 & 10 .\end{array}$ $\begin{array}{llllllllllllllll}1691 & 1562 & 790 & 718 & 1486 & 1179 & 2539 & 2640 & 667 & 432 & 471 & 684 \% & 1003 & 1254 & 1299\end{array}$ $\begin{array}{lllllllllllllll}106 & 104 & 113 & 103 & 106 & 107 & 102 & 110 & 83 & 108 & 118 & 114 & 111 & 114 & 130\end{array}$ $\begin{array}{lllllllllllllll}12 & 10 & 4 & 5 & 11 & 8 & 19 & 18 & 5 & 1 & 1 & 2 & 4 & 6 & 7\end{array}$ $\begin{array}{lllllllllllllll}1331 & 1152 & 483 & 519 & 1179 & 900 & 2100 & 2077 & 476 & 149 & 149 & 284 & 533 & 761 & 921\end{array}$ \begin{tabular}{lllllllllllllll}
111 & 115 & 121 & 104 & 107 & 113 & 111 & 115 & 95 & 149 & 149 & 142 & 133 & 127 & 132 \\
\hline
\end{tabular} 
Ces résultats sont corroborés par les Indices Trophiques moyens totaux qui classent les cours d'eau dans le même ordre (Fig. 3). Ils traduisent également assez bien la géologie, et, par là-même, le paramètre intégrateur de la conductivité, sans que la relation linéaire soit très étroite en raison des sauts quantitatifs de la conductivité entre les cours d'eau.

Les résultats du Diagnostic Phytoécologique sont décevants, les classements pour le pH et le sel aboutissant à la seule différenciation entre les cours d'eau acides et calcaires, ceux pour l'azote n'étant pas interprétables.

Le Taux de Dommage ne permet pas de telles différenciations des cours d'eau, en raison de la référence à des phytocénoses qui intègrent dès le départ la géologie et la trophie des eaux.

Les corrélations entre la conductivité et les indices macrophytes, donnent des coefficients $r^{2}$ de 0,38 pour le Taux de Dommage, 0,45 pour le Score Index par Taxon, 0,51 et 0,56 pour les Indices Trophiques moyens totaux et des sténoèces.

\subsubsection{Les variations amont-aval d'indices}

Pour la Moselotte et la Noye, on observe globalement une diminuation du Score Index Par Taxon de l'amont vers l'aval, traduisant ce que Muller (1990) appelle la « séquence d'eutrophisation ».

Des chutes de Score Index Par Taxon marquent les pollutions graves : la papeterie sur l'Avre (baisse de 4,67 en moyenne de $\mathrm{A} 1 \mathrm{M}$ et $\mathrm{A} 1 \mathrm{~V}$, à 2,67 en $\mathrm{A} 2$ ), la station d'épuration sur la Noye $(4,73$ en moyenne sur N1 à 4,27 en N2), les premiers rejets urbains sur la Moselotte (8,00 en M2, 5,50 en M3). Sur la Petite Seille, l'influence eutrophisante des étangs (augmentation de l'ammoniaque et des orthophosphates) se traduit par l'apparition de Potamogeton pectinatus dès la station PS2 et une diminution de l'indice (4,83 en PS1, 2,33 en PS2).

Le Taux de Dommage marque bien l'influence des étangs sur le Tilleul (T2) et la Petite Seille (PS2 et PS2B), mais aussi le recalibrage de l'amont de la Noye (N1M) qui entraîne une faible richesse spécifique.

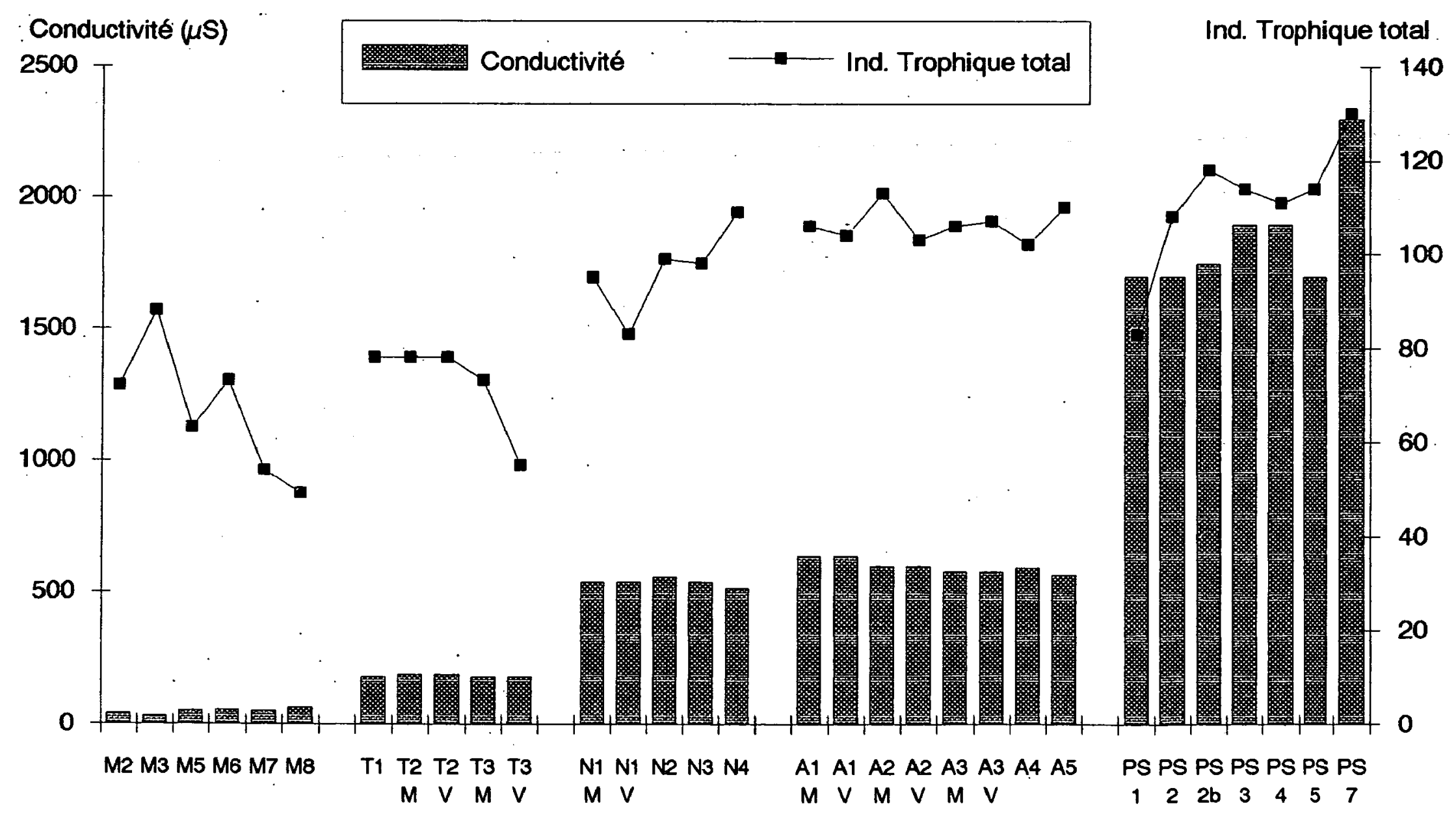

Fig. 3. Relation entre la conductivité et l'indice trophique. $-\mathbf{M}:$ Moselotte ; T : Tilleul ; N : Noye ; A : Avre ; PS : Petite Seille. Fig. 3. Relationship between conductivity and Trophic Index. $-M$ : Moselotte ; $T:$ Tilleul ; N : Noye ; A : Avre ; PS : Petite Seille. 
L'Indice Trophique moyen des sténoèces traduit l'eutrophisation par l'étang sur la Petite Seille (95 en PS1, 149 en PS2), les rejets sur la Moselotte amont (81 en $\mathrm{M} 2,110$ en M3) puis la récupération en aval (48 en M8).

\section{Discussion}

\subsection{Comparaison critique des indices et méthodes}

\subsubsection{La liste floristique}

Les listes floristiques dépendent des unités observées : celles intégrant la zone supra-aquatique sont bien évidemment plus riches que celles qui ne sont établies que sur le seul lit apparent (plus de 40 espèces pour le Tilleul, entre 4 et 15 pour les cours d'eau lorrains). La question de la place des hélophytes et bryophytes supra-aquatiques dans un système de bioindication de la qualité de l'eau se pose donc.

Du point de vue de l'acuité taxonomique, il est dommageable de confondre dans le diagnostic des espèces d'écologie fort différente, en regroupant l'ensemble des mousses, les callitriches, les renoncules aquatiques,...

Les listes d'espèces « autorisées " des différents auteurs amènent à se poser la question de leur pertinence (Luzula maxima considérée par Haslam), de leur exhaustivité (absence de Phragmites communis dans la liste de Harding), et de la prise en compte des espèces de ces listes lorsqu'elles se trouvent seulement en zone supra-aquatique.

De façon générale, les listes issues des indices d'Ellenberg semblent mieux adaptées aux zones humides qu'aux cours d'eau. Aussi, et bien que ces indices dissocient les facteurs écologiques, le diagnostic phytoécologique n'est pas à préconiser tant que de plus nombreuses espèces ne seront pas intégrées au système, dont des bryophytes et des algues.

\subsubsection{Le domaine de variation des résultats}

Les coefficients de variation des indices sont de 0,59 pour le Taux de Dommage, de 0,28 pour le Score Index Par Taxon, de 0,23 ou 0,32 pour les Indices Trophiques, mais de 0,10 pour l'azote dans le Diagnostic Phytoécologique selon Ellenberg, et de 0,15 pour le $\mathrm{pH}$. Au vu de ces résultats, le manque d'acuité du diagnostic phytoécologique avec les indices d'Ellenberg est manifeste.

De façon générale, lorsque la liste est restreinte, on renforce la variabilité : dans la méthode Newbold
\& Holmes, la seule prise en compte des espèces sténoèces donne un coefficient de variation de 0,32 , et celle de l'ensemble des macrophytes de 0,23 , ce qui correspond aux notes plus « moyennes 》 des espèces largement répandues.

\subsubsection{Pertinence et cohérence des indications}

Les indices de Harding, de Haslam, et de Newbold \& Holmes donnent des résultats interprétables, mettant en évidence différents niveaux de déséquilibre qui peuvent passer inaperçus avec des analyses d'eau trop ponctuelles ou mal situées dans le temps, comme, par exemple, l'influence des étangs sur le Tilleul : altérations de la qualité de l'eau, recalibrage, ombrage, ...

Toutefois, quelques problèmes de cohérence sont à signaler.

- Avec les méthodes Harding et Newbold \& Holmes, les phytocénoses dominées par les bryophytes apparaissent comme de meilleure qualité que celles composées de phanérogames, pour une même qualité d'eau, comme en témoignent par exemple les stations T2M (éclairée) et T2V (ombragée), ou T3M (abondance de phanérogames) et T3V (dominance des bryophytes).

- Les indices d'Ellenberg font apparaître des différences inexplicables au sein des stations, par exemple d'une unité de $\mathrm{pH}$ pour la liste simplifiée en A3. Là encore, compte-tenu du faible nombre d'espèces prises en compte, les régulations entre les notes des différentes espèces présentes au sein d'une même station ne peuvent s'effectuer.

- Le Taux de Dommage de Haslam intègre un indice de " changement de catégorie trophique ": on utilise dans le calcul un jugement sur ce que l'on veut en somme démontrer. Par ailleurs, une part d'appréciation est laissée à l'observateur puisqu'en général les espèces d'une station se répartissent dans plusieurs catégories trophiques : des critères strictement géochimiques seraient probablement préférables, même s'ils doivent être régionalisés.

\subsection{Quelles orientations pour une méthodologie intégratrice?}

\subsubsection{Compléter les listes des différents auteurs}

Les listes des différents auteurs étant incomplètes, il s'avère nécessaire de les enrichir au vu des recensements pratiqués en France et des synthèses existantes, notamment celle de Holmes (1983 in 
Standing Committee of Analysts (H.M.S.O.) 1987). Par ailleurs, compte-tenu des tendances écologiques des macroalgues et des bryophytes, qui diffèrent de celles des phanérogames, il est indispensable d'inclure au moins les plus représentatives dans ces listes.

\subsubsection{Vérifier l'écologie des espèces et donc la per-} tinence des indices qui leur sont attribués

La majorité des indices utilisés correspondent surtout à des jugements d'experts, à des études très localisées, ou à des analyses multidimensionnelles assez générales, sans que les relations statistiques entre les macrophytes et les différents paramètres de la qualité de l'eau aient été clairement établies, notamment par l'étude des profils écologiques.

L'approche d'Ellenberg est intéressante pour la distinction des facteurs qu'elle propose, même si une telle différenciation est artificielle. Il semblerait utile de compléter ses listes par les algues, les bryophytes et les hydrophytes, et pour deux facteurs au moins : la conductivité et les orthophosphates.

Par ailleurs, il faudra se poser la question de la stabilité du pouvoir bioindicateur des espèces selon les éco-régions (Haslam 1987, Haury \& Muller 1991). Il est probable que certaines populations ou certaines formes aient des valences écologiques différentes, ce qui mènerait soit à leur exclusion des indices macrophytes, soit à une «écorégionalisation » des indices, comparable à celle qui est en cours avec les invertébrés benthiques.

\subsubsection{Travailler à des niveaux complémentaires}

Plusieurs questions de complémentarité de niveaux d'étude se posent.

- Concernant la prise en compte des espèces supra-aquatiques, il s'avère que celles-ci masquent certaines différences liées à la qualité de l'eau. Il serait possible de les exclure totalement du diagnostic, mais alors on se priverait de possibilité de diagnostic dans les milieux à fonds instables (comme les fonds sableux, les torrents à galets, ...). Le calcul de deux indices, aquatique, d'une part, et intégrant la zone supra-aquatique, d'autre part, serait préférable.

- De la même façon, le problème de l'intégration des espèces euryèces dans les calculs synthétiques est délicat. Il semble intéressant de ne pas négliger dans les listes ces espèces $a$ priori très fréquentes en cours d'eau en même temps, leurs indices n'ont pas grande signification. C'est notamment le cas pour Agrostis stolonifera : sa présence fait chuter le Score Index Par Taxon, semble-t-il indûment.

- La prise en compte des recouvrements des espèces est complètement omise, si bien que les indices sont uniquement floristiques dans ces écosystèmes ouverts où les phénomènes de colonisation sont fréquents. Une pondération des présences par les recouvrements (Haury 1990a) s'avère souvent judicieuse pour porter un diagnostic.

- L'articulation des indices spécifiques avec l'analyse de la communauté est mal réalisée dans les méthodes élaborées jusqu'à présent. Le Taux de Dommage de Haslam est néanmoins un essai intéressant.

- Le point majeur, souligné par Haury (1990b) et dans les travaux du Laboratoire d'Ecologie de l'Université de Metz (Anonyme 1991), est la définition des phytocénoses de référence, qui reste encore à effectuer de façon conséquente, sur l'ensemble du territoire français. A ce niveau, les relations entre la phytosociologie et l'étude des ensembles floristiques restent à établir entre les spécialistes français.

\section{Conclusion}

Compte-tenu du développement précédent, il ressort que les macrophytes peuvent être utilisés comme des bioindicateurs de la qualité des cours d'eau, traduisant des paramètres de qualité des eaux, mais aussi comme témoins du milieu physique, notamment de la zonation longitudinale et des conditions d'éclairement.

Toutefois, les indices macrophytes s'ils sont applicables et donnent des résultats cohérents, sont encore du dqmaine de la recherche. Leur mise en œuvre se heurte encore à des obstacles méthodologiques (définition de l'unité d'étude, estimation des recouvrements, ...), taxonomiques (confusion d'espèces, niveau de détermination,...) et conceptuels (place des espèces euryèces dans les indices macrophytes, stabilité du pouvoir bioindicateur des espèces,...).

Dans un indice synthétique, la prise en compte des communautés devra être affinée, avec des coefficients de communauté analysant la structure des phytocénoses et comparant les végétations observées avec des phytocénoses de référence.

D'ores et déjà les méthodes existantes présentent une relative facilité de mise en œuvre qui est accueillie 
favorablement par des agents de terrain. Elles pourront ainsi compléter, avec toutes les réserves précitées; les outils de diagnostic de la qualité des cours d'eau.

\section{Remerciements}

Les auteurs remercient :

- N. Grasmuck (Université de Metz) qui a établi les listes floristiques des cours d'eau lorrains, sous la direction de S. Muller (Université de Metz) et de J. Haury, et effectué les analyses d'eau correspondantes ;

- J. Barbe (CEMAGREF Lyon) avec lequel les données picardes ont été récoltées et qui a participé à la détermination des algues ;

- les agents des Délégations Régionales 1 et 2 du Conseil Supérieur de la Pêche qui ont réalisé les analyses d'eau pour les cours d'eau picards et armoricains.

\section{Travaux cités}

Anonyme 1990. - Etude des végétaux fixés en relation avec la qualité du milieu. Laboratoire d'Ecologie, Univ. Metz (Coord.), ECOLOR, GEREEA, LOISIRS et DETENTE), rapp. Contr. Lab. Ecol. Univ. Metz -Agence de l'Eau RhinMeuse, 3 vol. : 94 p. + bibliogr., 64 p. ann., 17 p. référentiel.

Anonyme 1991. - Etude des végétaux fixés en relation avec la qualité du milieu - Note de synthèse. Laboratoire d'Ecologie, Univ. Metz (Coord.), ECOLOR, GEREEA, LOISIRS et DETENTE), rapp. Contr. Lab. Ecol. Univ. Metz - Agence de l'Eau Rhin-Meuse, 21 p.

Carbiener R., Trémolières M., Mercier J.L. \& Orcheit A. 1990. - Aquatic macrophyte communities as bioindicators of eutrophication in calcareous oligosaprobe stream waters (Upper Rhine plain, Alsace). Vegetatio, $86: 71-88$.

Codhant H., Valkman G., Haury J. \& Dutartre A. 1991. Les macrophytes aquatiques bioindicateurs de la qualité des eaux courantes - Département de la Lozère. Rapp. interm. Contr. CEMAGREF-INRA, Conseil Général. CEMAGREF Bordeaux et INRA Rennes, Conseil Général de Lozère, $146 \mathrm{p}$.

Standing Committee of Analysis (H.M.S.O.) 1987. - Methods for the use of aquatic macrophytes for assessing water quality 1985-86. Her Majesty's Stationery Office, London, 176 p. (with detailed accounts by Haslam S.M., Holmes N.T.H. \& Harding J.P.C.).

Ellenberg E. 1979. - Zeigerwerte der Gefässpflanzen von Mittleuropas, (3e éd.). Scripta Geobotanica $9: 122$ p.

Empain A., Lambinon J., Mouvet C. \& Kirchmann R. 1980. Utilisation des bryophytes aquatiques et subaquatiques comme indicateurs biologiques de la qualité des eaux courantes. In P. Pesson (Ed.) La pollution des eaux continentales - Incidences sur les biocénoses aquatiques ( $2^{e}$ éd.), Gauthier-Villars, Paris : 195-223.

Haslam S.M: 1978. - River plants. Cambridge Univ. Press, Cambridge, $396 \mathrm{p}$.

Haslam S.M. 1987. - River plants of Western Europe. Cambridge Univ. Press, Cambridge, 512 p.

Haslam S.M. \& Wolseley P.A. 1981. - River vegetation - Its identification, assessment and management. Cambridge Univ. Press, Cambridge, 154 p.
Haury J. 1985. - Etude écologique des macrophytes du Scorff (Bretagne-Sud). Thès. Doc.-Ing. Ecologie Univ. Rennes I, $243 \mathrm{p}$.

Haury J. 1989. - Diagnostic des cours d'eau à l'aide des macrophytes - Application à la Noye et l'Avre (80). Lab. Ecol. hydrobiol. INRA \& Ch. Botanique ENSA, Rennes, $29 \mathrm{p}$.

Haury J. 1990a. - Macrophytes du Trieux (Bretagne-Nord) : III : Relations macrophytes-qualité des eaux. Bull. Soc. Sc. nat. Ouest de la France, Nouv. Sér. 12 (4) : 141-154.

Haury J. 1990b. - Evaluation de la qualité de l'eau avec les macrophytes - Etude comparative de différents indices et méthodes. (Comm. Groupe Macrophytes A.N.P.P., Paris, 12/12/1989). Lab. Ecol. hydrobiol. INRA \& Ch. Botanique ENSA, Rennes, $13 \mathrm{p}$.

Haury J. 1991. - Diagnostic de deux cours d'eau picards à l'aide des macrophytes : l'Avre (80) et la Bresle (80-76). Lab. Ecol. hydrobiol. INRA \& Ch. Botanique ENSA, Rennes, $23 \mathrm{p}$.

Haury J. 1992. - Bilan de trois années d'étude. Observatoire des rivières dans le Parc Naturel Régional Normandie-Maine : la macrophytes - Rapp. synth. contr. P.N.R. NormandieMaine. Lab. Ecol. hydrobiol. INRA \& Ch. Botanique ENSA, Rennes, 104 p.

Haury J. \& Dutartre A. 1990. - Les macrophytes aquatiques : bioindicateurs de la qualité des eaux superficielles - Recommandations méthodologiques, Département de la Lozère. Lab. Ecol. hydrobiol. INRA \& Ch. Botanique ENSA, Rennes - CEMAGREF QEPP Cestas, Etude CEMAGREF n ${ }^{\circ} 68$ : $16 \mathrm{p}$.

Haury J. \& Muller S. 1991. - Variations écologiques et chrorologiques de la végétation macrophytique des rivières acides du Massif Armoricain et des Vosges du Nord (France). Rev. Sci. Eau 4 (4) : 463-482.

Holmes N.T.H. \& Whitton B.A. 1977. - The macrophytic vegetation of the river Tees : observed and predicted changes. Freshwat. Biol. 7 : 43-60.

Kohler A. 1975. - Submerse Makrophyten und ihre gesellschaften als Indikatoren der gewasserbelastung. Beitr. Naturk. Forsch. Sudwestdeutschl. 34 : 149-159.

Léglize L. \& Grasmuck N. 1992. - Etude des végétaux aquatiques des cours d'eau lorrains. Rapp. contr. Lab. Ecol. Univ. Metz - Agence de l'Eau Rhin-Meuse, 2 vol. : 42 p. + ann.

Léglize L. \& Peltre M.C. 1988. - Végétaux aquatiques fixés et eutrophisation du bassin amont de la Meuse - Cas du Vair (Eté 1987). Rapp. contr. Lab. Ecol. Univ. Metz - Agence de l'Eau Rhin-Meuse, 68 p. + ann.

Léglize L., Peltre M.C., Decloux J.P., Duval T., Paris P. \& Zumstein J.F., 1990. - Caractérisation des milieux aquatiques d'eaux courantes et végétation fixée. $14^{e}$ Conf. Int: COLUMA, Versailles 23-24 Janvier $1990:$ 237-245.

Mériaux J.L. 1982. - L'utilisation des macrophytes et des phytocénoses aquatiques comme indicateurs de la qualité des eaux. Naturalistes Belges, 63 : 18-24.

Mériaux J.L. \& Wattez J.R. 1980. - Les végétaux aquatiques et subaquatiques : relations avec la qualité des eaux. In P. Pesson (Ed.) La pollution des eaux continentales - Incidences sur les biocénoses aquatiques ( $2^{e}$ éd.), 225-242, GauthierVillars, Paris. 
Muller S. 1990. - Une séquence de groupements végétaux bioindicateurs d'eutrophisation croissante des cours d'eau faiblement minéralisés des Basses Vosges gréseuses du Nord. C.R. Acad. Sci. Paris, 310, Sér. III : 509-514.

Newbold C. \& Holmes N.T.H. 1987. - Nature conservation : water quality criteria and plants as water quality monitors. Water Pollution Control 86 (2) : 345-364.
Whitton B.A. 1979. - Plants as indicators of river water quality. In James A. \& Evison L. (Eds) Biological indicators of water quality, Wiley \& Sons Ed., Chichester : 5,1-5,34.

Wiegleb G. 1983. - Recherches méthodologiques sur les groupements végétaux des eaux courantes. In Géhu J.M. (Ed.) Les végétations aquatiqueś et amphibies, Colloq. Phytosociol. 10, Cramer, Vaduz : 69-83. 